\title{
Are night-time voiding and lower urinary tract symptoms significant risk factors for hip fractures caused by falling during the night in male subjects?
}

This article was published in the following Dove Press journal: Patient Preference and Adherence

\author{
Bogdan Hogea' \\ Razvan Bardan ${ }^{2}$ \\ Mihai Sandesc ${ }^{3}$ \\ Jenel Marian Patrascu J $\mathrm{r}^{3}$ \\ Alin Cumpanas ${ }^{2}$ \\ Bogdan Andor ${ }^{3}$ \\ 'Department of Anatomy, Victor Babes \\ University of Medicine and Pharmacy, \\ Timisoara, Romania; ${ }^{2}$ Department of \\ Urology, Victor Babes University of \\ Medicine and Pharmacy, Timisoara, \\ Romania; ${ }^{3}$ Second Department of \\ Orthopedics, Victor Babes University of \\ Medicine and Pharmacy, Timisoara, \\ Romania
}

Correspondence: Razvan Bardan Department of Urology, Victor Babes University of Medicine and Pharmacy, 156 Liviu Rebreanu Blvd., 300736 Timisoara, Romania

Tel +40723307888

Email razvan.bardan@gmail.com
Objectives: The aim of our study was to assess the incidence of night-time voiding in a population of male patients with hip fracture, and to analyze the correlations between the severity of the lower urinary symptoms and the other most significant comorbidities of the patients.

Patients and methods: We have initially selected a group of patients containing all males admitted into a Department of Orthopedic Surgery with the diagnosis of hip fracture, with indication for replacement surgery, over a four-year period. Applying well-defined inclusion and exclusion criteria, we have selected all the patients who have reported that falling during the night, on the way to the toilet (for micturition), was the event leading to the hip fracture. A comprehensive medical history and the International Prostate Symptom Score (IPSS) Questionnaire were gathered from all the patients and the obtained data were analyzed.

Results: From a total of 363 patients with hip fractures, 68 cases were attributable to nighttime voiding. The detailed analysis of the 68 patients has shown that all of them had significant nocturia (2.72 episodes of voiding per night, in average), but only 11 of them were already diagnosed with lower urinary tract symptoms (LUTS) due to benign prostatic hyperplasia (BPH). Moreover, only four of the eleven patients diagnosed with BPH were taking specific therapy at the moment of their falling leading to hip fracture. Subsequent analysis has shown that a group of 45 patients with no previously known co-morbidities, had similar IPSS score results with the total group of 68 patients.

Conclusions: Night-time voiding and LUTS are underdiagnosed in the general male population, as they represent significant risk factors for hip fractures due to falling during the night. The low level of BPH/LUTS therapy adherence adds a supplementary risk for falling during the night.

Keywords: hip fracture, night-time voiding, nocturia, low urinary tract symptoms, risk factors

\section{Introduction}

Nocturia, defined by the International Continence Society as the number of times urine is passed during the main sleep period, is a common cause of sleep interruption. Its incidence increases with age and is affecting both men and women. ${ }^{1,2}$ The most common causes of nocturia include excessive fluid intake during the evening, primary sleep disorders, diabetes mellitus, lower urinary tract obstruction due to benign prostatic hyperplasia, overactive bladder, cardiovascular disease, and congestive heart failure. ${ }^{1,3}$

Falls and subsequent fractures are important causes of morbidity and mortality among old age adults, with one-third of them having experienced at least one fall 
every year, and up to $20 \%$ of the falls resulting in fractures. $^{4-6}$ Some of the most frequent orthopedic incidents in these patients are represented by the hip fractures, with a significant rate of complications and mortality. ${ }^{7}$ It is globally estimated that hip fractures will affect around $18 \%$ of women and $6 \%$ of men during their lifetime; moreover, the global number of hip fractures is expected to increase to 4.5 million by the year 2050, with high material costs and difficult social and psychological rehabilitation. ${ }^{8}$ As a logical consequence, multiple studies have tried to identify and reduce the risk factors for falls and fractures. $^{9-12}$

The majority of the hip fractures (up to two-thirds) occur in female subjects, and most of them occur during daytime, only a quarter being recorded during the night. ${ }^{13}$ Since a patient with nocturia has to rise up from the bed and has to find his/her way to the toilet in low light or even total darkness, nocturia is considered a risk factor for falling, especially among the elderly. ${ }^{14-16}$ The relationship between nocturia and hip fractures was demonstrated by several longitudinal cohort studies, including those of Asplund, Temml et al, and Nagakawa et al. ${ }^{3,5,15}$ However, no study has tried yet to evaluate the impact of night-time voiding and lower urinary tract symptoms (LUTS) in correlation with the other known risk factors for falling and hip fractures.

\section{Objectives}

The main objectives of our prospective study were to assess the incidence of night-time voiding in a population of male patients with hip fracture, and to analyze the correlations between the severity of the lower urinary symptoms and the other most significant comorbidities of the patients.

\section{Materials and methods}

We have performed a prospective evaluation of all male patients admitted in a tertiary Department of Orthopedic Surgery, diagnosed with hip fracture with indication for hip replacement surgery, over a four-year period, between 01 January 2015 and 31 December 2018.

From this large cohort of male patients, we have selected those aged over 50 years, with good cognitive status, able to read and write, and without dementia or any other condition who could impair their level of understanding. During the mentioned period of time, a total number of 363 male patients with hip fractures were admitted into the hospital for hip replacement surgery.
Applying our selection criteria, we have found out that 328 were over 50 years of age, and 307 of them had good cognitive/consciousness status, making them suitable for our study.

During the initial interview with the patients, when taking the medical history, they were asked the following questions:

- What was the cause/circumstance of the traumatic event?

- If the cause of the fracture was falling from upright position, when did it happen? during the day or during the night?

From the total of 307 male patients, 282 have mentioned that it happened by falling from the standing position; 81 of the falls happened during the night, and 68 of these patients were on their way to the toilet, to empty their bladder, and were selected for our study. The initial patient selection process is presented in Figure 1.

The clinical study was approved by the Ethics Committee of the Timisoara Clinical Emergency County Hospital and was conducted in accordance with the Helsinki Declaration of Ethical Principles for Medical Research Involving Human Subjects. An informed consent form was presented to the 68 patients, and after signing it, they were included into the clinical study.

A complete medical history was recorded for all the patients, including the following data:

- The exact circumstances of the fall during the night, including the reason for being awake (eg, for going to the toilet);

- Level of vision;

- Capacity of independent walking;

- Previous history of diabetes mellitus, hypertension, cerebro-vascular disease, cardiac failure, chronic neurologic disease;

- Actual medication(s);

- Previous history of benign prostatic hyperplasia, or any visits to the urologist for voiding disorders, previous or actual medication for BPH (if applicable).

The patients have completed the International Prostate Symptom Score (IPSS), containing seven questions assessing the voiding function, and one question assessing the associated quality of life due to urinary symptoms, for the previous four weeks before the hip fracture. 


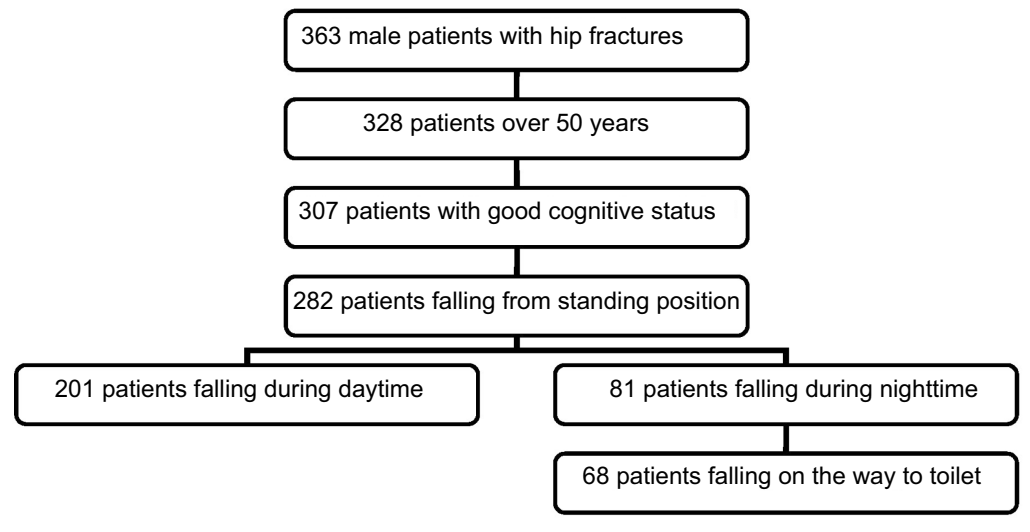

Figure I Flowchart representing the patient selection process.

The medical records of the patients were verified in the computerized database of the Hospital, corroborating with previous visits to the Urology Ambulatory, or with eventual hospitalizations in the Department of Urology, among other items.

All the gathered patient data were entered into a Microsoft Excel sheet, and statistical analysis was performed using the Data Analysis module of Microsoft Excel. For the determination of the statistical significance of our patient samples, we have generally performed the Welch's $t$-test, using the method of two-sample assuming unequal variances.

\section{Results}

In the studied time interval of four years (2015-2019), a total number of 1,161 patients were admitted in the Hospital diagnosed with hip fracture requiring hip replacement surgery; 798 patients were female and 363 were male, so the female/male ratio was 2.2 , and the male proportion was $31.3 \%$.

After the application of the above-mentioned selection criteria, we have obtained a group of 68 patients, so we may conclude that the incidence of hip fractures due to night-time voiding in our group of male patients was $18.7 \%$.

The average age of the group of 363 male patients with hip fracture was 70.3 years (range: $32-96$ years), while the average age of our selected group of 68 patients was 68.1 years (range: 50-91 years). Despite the fact that we have selected in our study group only patients older than 50 years, the difference between the two average age values could be explained by the exclusion of patients with dementia or impaired cognitive status, which were significantly older.

The main demographic and general information regarding our study group subjects are presented in Table 1. From the 68 analyzed patients, 33 had a good level of vision, not requiring glasses to move inside the house, and 55 had a good capacity of independent walking, not requiring help to move to the toilet.

The co-morbidity and current medication analysis gathered the results presented in Tables 2 and 3.

From Table 2 we can observe that cardiovascular disease and diabetes mellitus were the most frequent pathological conditions in this group of patients, as expected, while a significant proportion (22.1\%) were already diagnosed with prostatic disease, including benign prostatic hyperplasia (11 patients), prostate cancer (3), or chronic prostatitis (1).

Looking into the data from Table 3, we can find out that only four of the eleven patients with benign prostatic hyperplasia were taking specific drugs, respectively, three were under therapy with alpha-blockers as monotherapy, and one was under combined therapy, with an alpha blocker and a 5-alpha-reductase inhibitor.

Table I The main characteristics of the study group

\begin{tabular}{|l|l|l|}
\hline Number of subjects & $\mathbf{N}=\mathbf{6 8}$ \\
\hline Average age & 68.1 years (range: 50-91 years) & Absent: $45(66.2 \%)$ \\
\hline $\begin{array}{l}\text { Co-morbidities } \\
\text { Level of vision }\end{array}$ & Present: $23(33.8 \%)$ & Impaired: $35(51.5 \%)$ \\
Capacity of independent walking & Good: $33(48.5 \%)$ & Impaired: 13 (19.1\%) \\
\hline
\end{tabular}


Table 2 Principal co-morbidities of the patients from the study group

\begin{tabular}{|l|l|l|}
\hline Co-morbidity & N & $\%$ \\
\hline Hypertension & 13 & $19.1 \%$ \\
Diabetes mellitus & 6 & $8.8 \%$ \\
Chronic cardiac failure & 5 & $7.4 \%$ \\
Cerebro-vascular disease & 3 & $4.4 \%$ \\
Chronic renal failure & 3 & $4.4 \%$ \\
History of falling/fractures & 7 & $10.3 \%$ \\
Benign prostatic hyperplasia & II & $16.2 \%$ \\
Prostate cancer & 3 & $4.4 \%$ \\
Prostatitis & $\mathrm{I}$ & $1.5 \%$ \\
Overactive bladder & $\mathrm{I}$ & $1.5 \%$ \\
\hline
\end{tabular}

Table 3 The current medications taken by the patients from the study group

\begin{tabular}{|l|l|l|}
\hline Current medication & N & $\%$ \\
\hline No medication & 46 & $67.6 \%$ \\
Diuretics & 9 & $13.2 \%$ \\
Psychotropic drugs (including sedatives) & 7 & $10.3 \%$ \\
Drug therapy for diabetes mellitus & 6 & $8.8 \%$ \\
Alpha-blockers alone & 3 & $4.4 \%$ \\
Alpha-blockers and 5-alpha-reductase inhibitors & $\mathrm{I}$ & $1.5 \%$ \\
Anti-muscarinic agents & $\mathrm{I}$ & $1.5 \%$ \\
Beta-3-agonists & - & - \\
Anti-diuretics & - & - \\
\hline
\end{tabular}

In the following phase of our study, we have analyzed the results of the IPSS Questionnaire. Initially, we have calculated the average values, which are shown in Table 4.

From these results, we can observe that the average number of night-time micturition was 2.72, a quite high figure, while the average IPSS total score was 22.53, reflecting severe low urinary symptoms (the values corresponding for severe symptoms are in the range of 20-35). Moreover, the average quality of life question had a mean value of almost 4, meaning that the patients are mostly dissatisfied with their actual micturition status.

All eleven patients previously diagnosed with benign prostatic hyperplasia were initially prescribed specific drugs

Table 4 Results of the IPSS questionnaire

\begin{tabular}{|l|l|l|}
\hline Item & Average value & Standard Deviation \\
\hline Voiding sub-score & 12.76 & 2.35 \\
Question 7 - Nocturia & 2.72 & 0.96 \\
Storage sub-score & 9.96 & 2.00 \\
IPSS total score & 22.53 & 4.19 \\
Quality of life & 3.90 & 0.92 \\
\hline
\end{tabular}

by the urologists, including alpha-blockers. Unfortunately, only four of them were still under specific therapy at the moment of the fall leading to the hip fracture. The other seven have stopped their administration, with a worsening of their symptoms.

Although the sample size of the group of patients with benign prostatic hyperplasia was very small (11 patients), we compared the IPSS parameters for the patients still on specific therapy, and for those with no actual therapy. The results are presented in Table 5.

We can observe that all the differences between the average IPSS total score and sub-scores were statistically significant, confirming the efficacy of BPH therapy. Despite this success, these patients still had moderate nocturia, which in the end has led to falling during the night and to the subsequent hip fracture.

Following this, we have divided the 68 patients into four age groups (decades) and compared the average number of micturition episodes by night. The results are summed in Table 6.

The only age group with a significantly higher average number of micturitions per night was that of patients older than 80 years, which had to wake up for an average of 3.17 episodes per night, compared with the whole group, which had 2.72 episodes of voiding per night. In all the other studied age groups, the difference was not significant.

Finally, we have extracted from our group of 68 patients those with no previously diagnosed co-morbidities of any kind, and not taking any prescription drugs. We have obtained a group of 45 "clean" patients, and we have calculated the main IPSS parameters, trying to compare their results with the results of the whole group, containing also different confounding co-morbidities. The results are presented in Table 7.

The average age of the group of 45 patients with no comorbidities was 65.6 years, which was lower than the average age of the whole group of 68 patients (68.1 years); however, the difference was not statistically significant.

Regarding the results of the IPSS questionnaire, we can observe that the differences between all the average calculated sub-scores (voiding, nocturia, storage, quality of life, and total IPSSS) were quite similar, with no statistical significance between the two groups. This may lead us to the most important conclusion of our study, confirming that nocturia/night-time voiding and LUTS have significant impact as independent risk factors for falling during the night, leading to hip fractures. 
Table 5 Comparison between the patients under therapy for BPH and those with no therapy

\begin{tabular}{|l|l|l|l|}
\hline Group & Average value for patients treated for BPH & Average value for BPH patients with no therapy & $\begin{array}{l}\mathbf{p} \text { - } \\
\text { value }\end{array}$ \\
\hline Voiding sub-score & 6.5 & 15.57 & $<0.001$ \\
Question 7 - Nocturia & 1.5 & 3.43 & 0.001 \\
Storage sub-score & 8.0 & 12.86 & 0.003 \\
IPSS total score & 14.50 & 28.43 & $<0.001$ \\
Quality of life & 2.5 & 5.0 & $<0.001$ \\
\hline
\end{tabular}

Table 6 Relationship between age and night-time voiding

\begin{tabular}{|l|l|l|}
\hline Age group & Average micturition episodes/night & $p$-value \\
\hline Whole group & 2.72 & - \\
$50-59$ years & 2.73 & NS \\
$60-69$ years & 2.55 & NS \\
$70-79$ years & 2.67 & NS \\
$\geq 80$ years & 3.17 & 0.05 \\
\hline
\end{tabular}

\section{Discussion}

Previous studies have already confirmed that nocturia is an independent risk factor for falling and hip fractures, with a significantly higher (more than double) prevalence of hip fractures in patients with nocturia., ${ }^{3,5}$ This leads to high costs related to hospitalization, hip replacement surgery, social re-integration, and with increased mortality risk, too. ${ }^{17,18}$

Our study has confirmed the high incidence of lower tract urinary symptoms in a population with hip fractures, raising the need for earlier diagnosis, and effective treatment of these symptoms, generated usually by benign prostatic hyperplasia. Moreover, the low adherence to specific BPH therapy, which could be explained by a multitude of social and personal factors, including a subjectively perceived lack of efficacy, and the high price of the drug therapy, should be addressed, and new patient management strategies, generating more long-time therapy persistence, should be implemented.
The most important finding of our current research is that night-time voiding and LUTS have similar severity in patients with co-morbidities and patients with no previously diagnosed co-morbidity, so night-time voiding might represent an independent risk factor for hip fractures due to falling during the night in this patient population.

The main limitation of our study resides from the fact that it was performed in a group of patients already diagnosed with hip fracture, not allowing to gather clinical information regarding the real impact of night-time voiding on the risk of falling in the elderly population. However, in our knowledge, this study is the only one addressing the problem from another perspective, linking the risk of nocturnal falling with the severity of the lower urinary tract symptoms.

Another significant limitation is due to the fact that nocturnal polyuria was not correctly assessed in our patient population, being a more complex condition, difficult to assess (requires to measure the diuresis during the day and the night), and with a multitude of possible causes, including but not being limited to low nocturnal levels of vasopressin, congestive cardiac failure, obstructive sleep apnea, or chronic kidney disease. ${ }^{3,5,15,16}$

Moreover, the high levels of the IPSS score and subscores do not allow us to make a correct differentiation between the lower urinary tract symptoms for $\mathrm{BPH}$, and those found in patients with prostate cancer, chronic prostatitis, overactive bladder, and even neurogenic bladder.

Table 7 Comparison between the average IPSS sub-scores of the total group of patients with nocturia, and the group of patients with no previously diagnosed co-morbidities

\begin{tabular}{|l|l|l|l|}
\hline & Total group of 68 patients & Group of 45 “clean" patients & p-value \\
\hline Voiding sub-score & 12.76 & 12.82 & NS \\
Question 7 - Nocturia & 2.72 & 2.62 & NS \\
Storage sub-score & 9.96 & 9.47 & NS \\
IPSS total score & 22.53 & 22.29 & NS \\
Quality of life & 3.9 & 3.76 & NS \\
\hline
\end{tabular}


Although all the evaluated patients had their main sleep period during the night, our questionnaire has not included, unfortunately, more specific data regarding nocturia: we did not record information regarding the number of the nocturnal voiding at the moment of falling, which should have been valuable, in addition to the average number of micturitions/night, assessed by the IPSS questionnaire. We also did not record the exact time at the time of the falling and the number of hours of uninterrupted sleep until that moment.

Despite the acceptable size of our initial cohort, of 363 patients, due to applying strict inclusion criteria, we had a final patient population of 68 patients, which is relatively small, reducing the representativity of our results. However, we have obtained very interesting results, with statistical significance, which need to be validated on larger patient populations, from more centers.

\section{Conclusions}

Night-time voiding and low urinary tract symptoms are underdiagnosed in the general male population, as they represent significant risk factors for hip fractures due to falling during the night. The low level of BPH/LUTS therapy adherence represents a supplementary risk for falling during the night. Future prospective studies should find whether the appropriate long-term therapy of LUTS due to $\mathrm{BPH}$ reduces the risk of hip fractures, and the subsequent morbidity and mortality risks.

\section{Disclosure}

The authors report no conflicts of interest in this work.

\section{References}

1. Abrams P, Cardozo L, Wagg A, Wein A, editors. Incontinence 6th Edition (2017). International Continence Society, Bristol UK. ISBN: 978-0956960733.

2. D'Ancona C, Haylen B, Oelke M, et al. The International Continence Society (ICS) report on the terminology for adult male lower urinary tract and pelvic floor symptoms and dysfunction. Neurourol Urodyn. 2019;38(2):433-477. doi:10.1002/nau.23897
3. Nakagawa H, Niu K, Hozawa A, et al. Impact of nocturia on bone fracture and mortality in older individuals: a Japanese longitudinal cohort study. $J$ Urol. 2010;184:1413-1418. doi:10.1016/j.juro.2010.05.093

4. Stewart RB, Moore MT, May FE, Marks RG, Hale WE. Nocturia: a risk factor for falls in the elderly. $J$ Am Geriatr Soc. 1992;40:12171220.

5. Asplund R. Hip fractures, nocturia, and nocturnal polyuria in the elderly. Arch Gerontol Geriatr. 2006;43:319-326. doi:10.1016/j. archger.2005.12.002

6. Szabo SM, Gooch KL, Walker DR, Johnston KM, Wagg AS. The association between overactive bladder and falls and fractures: a systematic review. Adv Ther. 2018;35:1831-1841. doi:10.1007/ s12325-018-0796-8

7. Florence CS, Bergen G, Atherly A, Burns E, Stevens J, Drake C. Medical costs of fatal and nonfatal falls in older adults. $J$ Am Geriatr Soc. 2018;66:693-698. doi:10.1111/jgs.15304

8. Veronese N, Maggi S. Epidemiology and social costs of hip fracture. Injury. 2018;49(8):1458-1460. doi:10.1016/j.injury. 2 018.04 .015

9. De Andrea S, Lucenteforte E, Bravi F, Foschi R, La Vecchia C, Negri E. Risk factors for falls in community-dwelling older people: a systematic review and meta-analysis. Epidemiology. 2010;21:658668. doi:10.1097/EDE.0b013e3181e89905

10. Tinetti ME, Speechley M, Ginter SF. Risk factors for falls among elderly persons living in the community. $N$ Engl $J$ Med. 1988;319:1701-1707. doi:10.1056/NEJM198812293192604

11. Patrascu JM, Vermesan D, Mioc ML, et al. Musculo-skeletal tumors incidence and surgical treatment - a single center 5-year retrospective. Eur Rev Med Pharmacol Sci. 2014;18:3898-3901.

12. Luukinen H, Koski K, Laippala P, Kivelä SL. Factors predicting fractures during falling impacts among home-dwelling older adults. J Am Geriatr Soc. 1997;45:1302-1309.

13. Leavy B, Aberg AC, Melhus H, Mallmin H, Michaëlsson K, Byberg L. When and where do hip fractures occur? A population-based study. Osteoporos Int. 2013;24(9):2387-2396. doi:10.1007/s00198013-2333-6

14. Galizia G, Langellotto A, Cacciatore F, et al. Association between nocturia and falls-related long-term mortality risk in the elderly. $\mathrm{J} \mathrm{Am}$ Med Dir Assoc. 2012;13:640-644. doi:10.1016/j.jamda.2012.05.016

15. Temml C, Ponholzer A, Gutjahr G, Berger I, Marszalek M, Madersbacher S. Nocturia is an age-independent risk factor for hipfractures in men. Neurourol Urodyn. 2009;28:949-952. doi:10.1002/ nau. 20712

16. Vaughan CP, Brown CJ, Goode PS, Burgio KL, Allman RM, Johnson TM. The association of nocturia with incident falls in an elderly community-dwelling cohort. Int J Clin Pract. 2010;64:577-583. doi:10.1111/j.1742-1241.2009.02326.x

17. Noguchi N, Chan L, Cumming RG, et al. A systematic review of the association between lower urinary tract symptoms and falls, injuries, and fractures in community-dwelling older men. Aging Male. 2016;19(3):168-174. doi:10.3109/13685538.2016.1169399

18. Parsons JK, Mougey J, Lambert L, et al. Lower urinary tract symptoms increase the risk of falls in older men. BJU Int. 2009;104:63-68. doi:10.1111/j.1464-410X.2008.08317.x 


\section{Publish your work in this journal}

Patient Preference and Adherence is an international, peer-reviewed, open access journal that focusing on the growing importance of patient preference and adherence throughout the therapeutic continuum. Patient satisfaction, acceptability, quality of life, compliance, persistence and their role in developing new therapeutic modalities and compounds to optimize clinical outcomes for existing disease

states are major areas of interest for the journal. This journal has been accepted for indexing on PubMed Central. The manuscript management system is completely online and includes a very quick and fair peer-review system, which is all easy to use. Visit http:// www.dovepress.com/testimonials.php to read real quotes from published authors. 